\title{
Structural and evolutionary insights point to allosteric regulation of TRP ion channels
}

Jacob K. Hilton ${ }^{1,2}$, Minjoo Kim ${ }^{1,2}$, and Wade D. Van Horn ${ }^{1,2 *}$

${ }^{1}$ School of Molecular Sciences, Arizona State University, Tempe, AZ. 85287

${ }^{2}$ The Biodesign Institute Centers for Personalized Diagnostics and Mechanisms of Evolution, Arizona State University, Tempe, AZ. 85281

*Corresponding Author: wade.van.horn@asu.edu

Table S1. Percent identity matrix for human TRP channels. Clustal Omega was used to generate a multiple sequence alignment and percent identity matrix of the 27 human TRP channels and the Drosophila channel TRPN1. Colors correspond to the colors of TRP channel subfamilies shown in Figure 1 and highlight sequence identity within each subfamily.

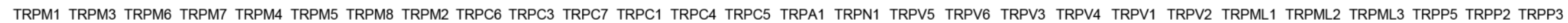

\begin{tabular}{|c|c|c|c|c|c|c|c|c|c|c|c|c|c|c|c|c|c|c|c|c|c|c|c|c|c|c|c|c|}
\hline TRPM1 & 100.0 & 64.6 & 47.1 & 49.5 & 30.1 & 29.4 & 29.2 & 31.3 & 19.4 & 17.6 & 17.8 & 17.0 & 17.9 & 16.7 & 12.7 & 15.6 & 14.6 & 13.3 & 15.4 & 13.4 & 14.5 & 16.0 & 8.9 & 9.3 & 8.8 & 12.4 & 14.0 & 14.4 \\
\hline TRPM3 & - & 100.0 & 48.2 & 53.5 & 29.8 & 29.4 & 29.9 & 30.7 & 18.7 & 16.5 & 17.8 & 17.2 & 17.7 & 17.8 & 13.1 & 15.0 & 14.5 & 12.9 & 15.4 & 13.4 & 15.6 & 16.5 & 8.6 & 9.4 & 8.3 & 12.4 & 13.3 & 12.6 \\
\hline TRPM6 & - & - & 100.0 & 57.1 & 28.3 & 28.3 & 26.7 & 28.4 & 19.5 & 17.7 & 18.8 & 15.8 & 18.6 & 17.1 & 12.6 & 14.2 & 13.2 & 12.8 & 15.3 & 12.3 & 13.0 & 14.6 & 9.9 & 8.7 & 9.1 & 11.6 & 12.9 & 12.0 \\
\hline TRPM7 & - & - & - & 100.0 & 28.3 & 26.9 & 28.3 & 29.5 & 18.8 & 16.3 & 17.3 & 16.0 & 17.4 & 17.4 & 11.9 & 14.7 & 13.0 & 12.3 & 13.5 & 11.9 & 13.0 & 15.0 & 11.0 & 9.6 & 10.5 & 12.3 & 12.5 & 11.1 \\
\hline TRPM4 & - & - & - & - & 100.0 & 46.4 & 34.0 & 37.7 & 18.3 & 18.6 & 17.9 & 17.8 & 19.6 & 18.8 & 13.0 & 16.4 & 13.2 & 13.8 & 15.2 & 13.8 & 13.8 & 14.8 & 10.2 & 8.3 & 10.0 & 10.6 & 14.3 & 11.1 \\
\hline TRPM5 & - & - & - & - & - & 100.0 & 35.6 & 39.5 & 18.3 & 17.9 & 17.9 & 16.4 & 19.3 & 19.1 & 12.8 & 15.6 & 13.2 & 12.7 & 14.0 & 14.8 & 12.9 & 14.0 & 10.0 & 8.3 & 10.8 & 10.9 & 11.7 & 11.7 \\
\hline TRPM8 & - & - & - & - & - & - & 100.0 & 42.6 & 18.0 & 18.3 & 18.0 & 17.5 & 20.3 & 19.3 & 13.6 & 15.0 & 12.7 & 13.1 & 14.3 & 13.6 & 14.3 & 15.7 & 10.2 & 8.0 & 9.7 & 11.9 & 14.3 & 12.5 \\
\hline TRPM2 & - & - & - & - & - & - & - & 100.0 & 21.2 & 20.8 & 20.8 & 19.9 & 18.6 & 19.2 & 12.7 & 16.4 & 12.8 & 11.7 & 14.1 & 13.3 & 12.3 & 14.4 & 9.5 & 8.1 & 8.8 & 11.2 & 12.9 & 13.4 \\
\hline TRPC6 & - & - & - & - & - & - & - & - & 100.0 & 74.7 & 74.9 & 36.2 & 40.8 & 39.5 & 16.2 & 21.7 & 13.5 & 14.0 & 14.0 & 14.6 & 14.2 & 14.9 & 11.5 & 12.1 & 12.9 & 17.4 & 16.7 & 17.2 \\
\hline TRPC3 & - & - & - & - & - & - & - & - & - & 100.0 & 81.4 & 35.7 & 42.0 & 42.1 & 15.0 & 22.8 & 13.1 & 13.1 & 14.5 & 14.4 & 14.4 & 14.5 & 12.7 & 12.9 & 13.5 & 16.5 & 15.1 & 17.7 \\
\hline TRPC7 & - & - & - & - & - & - & - & - & - & - & 100.0 & 34.8 & 41.6 & 40.5 & 14.4 & 21.1 & 12.8 & 13.0 & 14.0 & 14.3 & 13.9 & 14.0 & 12.0 & 12.3 & 13.4 & 16.3 & 16.5 & 17.8 \\
\hline TRPC1 & - & - & - & - & - & - & - & - & - & - & - & 100.0 & 46.5 & 45.5 & 12.2 & 20.2 & 12.8 & 13.5 & 14.1 & 12.4 & 14.3 & 13.6 & 11.5 & 11.7 & 10.4 & 10.9 & 10.0 & 11.9 \\
\hline TRPC4 & - & - & - & - & - & - & - & - & - & - & - & - & 100.0 & 68.3 & 15.1 & 21.7 & 16.2 & 16.4 & 16.3 & 14.4 & 16.6 & 16.0 & 12.8 & 10.9 & 11.7 & 13.4 & 13.5 & 15.9 \\
\hline TRPC5 & - & - & - & - & - & - & - & - & - & - & - & - & - & 100.0 & 13.5 & 21.6 & 15.0 & 15.2 & 16.5 & 14.4 & 16.0 & 16.3 & 13.0 & 12.5 & 11.9 & 12.7 & 13.5 & 15.1 \\
\hline TRPA1 & - & - & - & - & - & - & - & - & - & - & - & - & - & - & 100.0 & 22.9 & 18.2 & 19.2 & 18.5 & 18.5 & 18.4 & 18.2 & 15.7 & 13.6 & 14.7 & 16.7 & 16.4 & 15.8 \\
\hline TRPN1 & - & - & - & - & - & - & - & - & - & - & - & - & - & - & - & 100.0 & 20.3 & 19.7 & 19.2 & 18.5 & 20.0 & 20.4 & 12.4 & 12.7 & 13.0 & 15.1 & 14.1 & 13.1 \\
\hline TRPV5 & - & - & - & - & - & - & - & - & - & - & - & - & - & - & - & - & 100.0 & 74.7 & 28.3 & 29.5 & 28.2 & 27.5 & 15.1 & 15.6 & 16.5 & 13.4 & 15.4 & 14.6 \\
\hline TRPV6 & - & - & - & - & - & - & - & - & - & - & - & - & - & - & - & - & - & 100.0 & 27.8 & 29.4 & 28.4 & 28.0 & 15.6 & 15.4 & 16.5 & 13.5 & 14.3 & 13.7 \\
\hline TRPV3 & - & - & - & - & - & - & - & - & - & - & - & - & - & - & - & - & - & - & 100.0 & 41.5 & 41.9 & 38.2 & 13.7 & 14.4 & 13.5 & 13.8 & 14.4 & 15.7 \\
\hline TRPV4 & - & - & - & - & - & - & - & - & - & - & - & - & - & - & - & - & - & - & - & 100.0 & 45.6 & 43.2 & 13.7 & 14.4 & 14.6 & 16.9 & 15.8 & 17.0 \\
\hline TRPV1 & - & - & - & - & - & - & - & - & - & - & - & - & - & - & - & - & - & - & - & - & 100.0 & 46.8 & 14.3 & 13.7 & 13.0 & 13.1 & 13.4 & 13.5 \\
\hline TRPV2 & - & - & - & - & - & - & - & - & - & - & - & - & - & - & - & - & - & - & - & - & - & 100.0 & 16.1 & 16.0 & 15.6 & 11.2 & 12.7 & 14.0 \\
\hline RPML1 & - & - & - & - & - & - & - & - & - & - & - & - & - & - & - & - & - & - & - & - & - & - & 100.0 & 48.7 & 56.4 & 17.6 & 17.9 & 18.9 \\
\hline RPML2 & - & - & - & - & - & - & - & - & - & - & - & - & - & - & - & - & - & - & - & - & - & - & - & 100.0 & 57.1 & 18.0 & 17.1 & 17.6 \\
\hline TRPML3 & - & - & - & - & - & - & - & - & - & - & - & - & - & - & - & - & - & - & - & - & - & - & - & - & 100.0 & 19.2 & 19.6 & 17.3 \\
\hline TRPP5 & - & - & - & - & - & - & - & - & - & - & - & - & - & - & - & - & - & - & - & - & - & - & - & - & - & 100.0 & 47.0 & 46.9 \\
\hline TRPP2 & - & - & - & - & - & - & - & - & - & - & - & - & - & - & - & - & - & - & - & - & - & - & - & - & - & - & 100.0 & 50.8 \\
\hline TRPP3 & - & - & - & - & - & - & - & - & - & - & - & - & - & - & - & - & - & - & - & - & - & - & - & - & - & - & - & 100.0 \\
\hline
\end{tabular}


Table S2. Percent identity matrix for human TRP channels from the same multiple sequence alignment described in Table S1. In this case, the color intensity from white to red illustrates increasing sequence conservation.

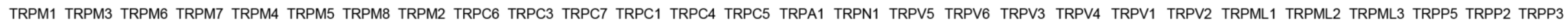

\begin{tabular}{|c|c|c|c|c|c|c|c|c|c|c|c|c|c|c|c|c|c|c|c|c|c|c|c|c|c|c|c|c|}
\hline PPM1 & 0.0 & 6 & .1 & 49.5 & 0.1 & 9.4 & 29.2 & 1.3 & 9.4 & 17.6 & 17.8 & 17.0 & 17.9 & 6.7 & 12.7 & 15.6 & 14.6 & 13.3 & 15.4 & 13.4 & 14.5 & 16.0 & 3.9 & 10 & 8 & 2.4 & 14.0 & 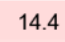 \\
\hline TRPM3 & 64.6 & 100.0 & 8.2 & 3.5 & 29.8 & 9.4 & 29.9 & 0.7 & 8.7 & 16.5 & 17.8 & 17.2 & 17.7 & 7.8 & 13.1 & 15.0 & 14.5 & 12.9 & 15.4 & 13.4 & 15.6 & 16.5 & 8.6 & 9.4 & 8.3 & 2.4 & 13.3 & 12.6 \\
\hline TRPM6 & 47.1 & 48.2 & 100.0 & 7.1 & 28.3 & 28.3 & & & & 17.7 & 18.8 & & 18.6 & & 12.6 & & & 12.8 & & 12.3 & 13.0 & 4.6 & 9.9 & 8.7 & 9.1 & 1.6 & 2.9 & 2.0 \\
\hline RPM7 & 49.5 & 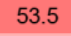 & .1 & 0.0 & 3 & 3.9 & 3 & & & 3 & 7.3 & 0.0 & 17 & 7.4 & 1.9 & & & 2.3 & 3.5 & 11.9 & 13.0 & 15.0 & 11.0 & 6 & 10.5 & 2.3 & 2.5 & 1.1 \\
\hline TRPM4 & 30.1 & 29.8 & 28.3 & 28.3 & 100.0 & 6.4 & .0 & 7.7 & 3.3 & 18.6 & 7.9 & 17.8 & 19.6 & 18.8 & 13.0 & 16.4 & 3.2 & 13.8 & 15.2 & 13.8 & 13.8 & 14.8 & 10.2 & 3 & 10.0 & 10.6 & 14.3 & 11.1 \\
\hline TRPM & 29.4 & 29.4 & 28.3 & 26.9 & 46.4 & 100.0 & 35.6 & & 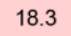 & 17.9 & 17.9 & 16.4 & 19.3 & 19.1 & 12.8 & & & 12.7 & & 14.8 & 12.9 & 4.0 & 10.0 & 3 & 0.8 & 0.9 & 1.7 & 1.7 \\
\hline RPN & 29.2 & 29.9 & 26.7 & 28.3 & 1.0 & 5.6 & 00.0 & 6 & 8.0 & 18.3 & 18.0 & 17.5 & 20.3 & 19.3 & 13.6 & 18 & 2.7 & 13.1 & 3 & 13.6 & 14.3 & 15.7 & 10.2 & 0 & 9.7 & 11.9 & 14.3 & 12.5 \\
\hline TRPM2 & 31.3 & 30.7 & 28.4 & 29.5 & 37.7 & 39.5 & 42.6 & 100.0 & 21.2 & 20.8 & 20.8 & 19.9 & 18.6 & 19.2 & 12.7 & 16.4 & 12.8 & 11.7 & 14.1 & 13.3 & 12.3 & 14.4 & 9.5 & 8.1 & 8.8 & 11.2 & 12.9 & 13.4 \\
\hline TRPC6 & 19.4 & 18 & 19.5 & 18.8 & 18.3 & 3.3 & 18.0 & & 00.0 & 74.7 & 74.9 & 36.2 & 40.8 & 39.5 & 16.2 & 21.7 & 13.5 & 14.0 & 1.0 & 14.6 & 14.2 & 14.9 & 1.5 & 12.1 & 2.9 & 7.4 & 16.7 & 7.2 \\
\hline RPC & 17.6 & 16. & 17. & 16.3 & 8. & .9 & 8. & 0.8 & 4.7 & 100.0 & 81.4 & 35.7 & 42.0 & 42.1 & 15.0 & 2.8 & & 13.1 & 4.5 & 14.4 & 14.4 & 14.5 & 12.7 & 2. & 3.5 & .5 & 15.1 & 17.7 \\
\hline TRPC7 & 17.8 & 17.8 & 18.8 & 17.3 & 17.9 & 17.9 & 18.0 & 0.8 & 74.9 & 81.4 & 100.0 & 34.8 & 41.6 & 40.5 & 14.4 & 21.1 & 12.8 & 13.0 & 14.0 & 14.3 & 13.9 & 14.0 & 12.0 & 12.3 & 13.4 & 16.3 & 16.5 & 17.8 \\
\hline TRPC1 & 17.0 & 17.2 & 15.8 & 16.0 & 1 & 16.4 & 17.5 & 1 & 36.2 & 35.7 & 34.8 & 100.0 & 46.5 & 45.5 & 12.2 & 20.2 & 12.8 & 13.5 & 14.1 & 12.4 & 14.3 & 13.6 & 11.5 & 11.7 & 10.4 & 0.9 & 10.0 & 11.9 \\
\hline RPC4 & 17.9 & 17.7 & 18.6 & 17.4 & 19.6 & 19.3 & 20 & 8.6 & 0.8 & 2 & 41. & 46.5 & 100.0 & 68.3 & 15.1 & 21.7 & 16.2 & 16.4 & 16.3 & 14.4 & 16.6 & 16.0 & 12 & 10 & 11.7 & 13.4 & 13.5 & 15.9 \\
\hline TRPC5 & 16.7 & 17.8 & 17.1 & 17.4 & 18.8 & 19.1 & 19.3 & 19.2 & 39.5 & 42.1 & 40.5 & 45.5 & 68.3 & 100.0 & 13.5 & 21.6 & 15.0 & 15.2 & 16.5 & 14.4 & 16.0 & 16.3 & 13.0 & 12.5 & 11.9 & 12.7 & 13.5 & 15.1 \\
\hline TRPA & 12.7 & 1 & 12.6 & 11.9 & & 12.8 & 13 & & 0.2 & & 1. & 12.2 & 1 & 5 & 100.0 & 22.9 & 18.2 & 1 & & 18.5 & 18.4 & 8.2 & 15.7 & 13.6 & 7 & 3.7 & 8.4 & 5.8 \\
\hline IRPN1 & 15.6 & 15.0 & 14.2 & 14.7 & 16.4 & 15.6 & 15.0 & 6.4 & 1.7 & 22. & 21.1 & 20.2 & 21.7 & 21.6 & 22.9 & 100.0 & 20.3 & 19.7 & 9.2 & 18.5 & 20.0 & 20.4 & 12.4 & 12.7 & 13.0 & 15.1 & 14.1 & 13.1 \\
\hline TRPV5 & 14.6 & 14.5 & 13.2 & 13.0 & 13.2 & 13.2 & 12.7 & 2.8 & 3.5 & 13.1 & 12.8 & 12.8 & 16.2 & 5.0 & 18.2 & 20.3 & 100.0 & 74.7 & 28.3 & 29.5 & 28.2 & 27.5 & 15.1 & 15.6 & 16.5 & 13.4 & 15.4 & 14.6 \\
\hline RPV6 & 13.3 & 12 & 1 & & & 1 & & & & 1 & & 13.5 & 1 & & 19 & 19.7 & 74.7 & 100.0 & 27.8 & 29.4 & 28.4 & 28.0 & 15 & 15 & 6.5 & .5 & 3 & 13.7 \\
\hline TRPV3 & 15.4 & 15.4 & 15.3 & 13.5 & 15.2 & 4.0 & 14 & & 4.0 & 14.5 & 14 & 14.1 & 16.3 & 16.5 & 18.5 & 19.2 & 28.3 & 27.8 & 100.0 & 41.5 & 41.9 & 38.2 & 13.7 & 14.4 & 13.5 & 13.8 & 14.4 & 15.7 \\
\hline TRPV4 & 13.4 & 13.4 & 12.3 & 11.9 & 13.8 & 14.8 & 13.6 & 3 & 4.6 & 14.4 & 4.3 & 12.4 & 14.4 & 4.4 & 18.5 & 18.5 & 9.5 & 29.4 & 41.5 & 100.0 & 45.6 & 43.2 & 13.7 & 14.4 & 14.6 & 16.9 & 15.8 & 17.0 \\
\hline TRPV1 & 14.5 & 15 & 13.0 & 13. & & 12.9 & & & & 14 & & & 1 & 16.0 & 18 & & & 28 & .9 & 45.6 & 100.0 & 46.8 & 4.3 & 13.7 & 13.0 & 3.1 & 13.4 & 13.5 \\
\hline RPV2 & 16.0 & 16.5 & 14.6 & 15.0 & 14.8 & 14.0 & 15.7 & 14.4 & 4.9 & 14.5 & 14.0 & 13.6 & 16.0 & 16.3 & 18.2 & 20.4 & 27.5 & 28.0 & 38.2 & 43.2 & 46.8 & 100.0 & 16.1 & 16.0 & 15.6 & 11.2 & 12.7 & 14.0 \\
\hline 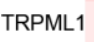 & 8.9 & 8.6 & 9.9 & 11.0 & 10.2 & 10.0 & 10.2 & . & 1.5 & 12.7 & 12.0 & 11.5 & 12.8 & 0 & 15.7 & 12.4 & 1 & 15.6 & 13.7 & 13.7 & 14.3 & 16.1 & 100.0 & 48.7 & 6.4 & 17.6 & 17.9 & 18.9 \\
\hline PPML & 9.3 & 9 & 8.7 & 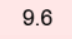 & 0.0 & & & & 12.1 & 12 & 12 & 11.7 & 1 & 16 & 10 & 12.1 & 15.6 & 10 & & 14.4 & 73.1 & 16.0 & 48.7 & 100.0 & 57.1 & 18.0 & 17.1 & 17.6 \\
\hline RPML3 & 8.8 & 8.3 & 9. & 10.5 & 10.0 & 10.8 & & 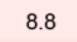 & 12.9 & 13.5 & 13 & 10 & .... & 11.9 & 14 & 13.0 & 16.5 & 16 & & 14.6 & 13 & 15.6 & 56.4 & 57.1 & 100.0 & 19.2 & 19.6 & 17.3 \\
\hline TRP & 12.4 & 12.4 & 11.6 & 12.3 & 10.6 & 10.9 & 11.9 & 1.2 & 7.4 & 16.5 & 16.3 & 10.9 & & 2.7 & 16.7 & 15.1 & 3.4 & 13.5 & 13.8 & 16.9 & 13.1 & 1.2 & 7.6 & 18.0 & 9.2 & 100.0 & 47.0 & 46.9 \\
\hline & 14.0 & 13. & 12.9 & 12.5 & 14 & 1 & 14 & & & 15 & 16 & 10 & 1 & 13 & 16 & 1 & 1 & & & 15.8 & 13 & 12.7 & 17.9 & 17.1 & 19.6 & 47.0 & 100.0 & 50.8 \\
\hline & 14.4 & 12.6 & 0 & & & & & & & 17.7 & & 8 & 15.9 & T. & 15.8 & 13.1 & 6 & 13.7 & 15 & 17.0 & 13. & 14.0 & 18.9 & 17.6 & 17.3 & 46.9 & 50.8 & 100. \\
\hline
\end{tabular}



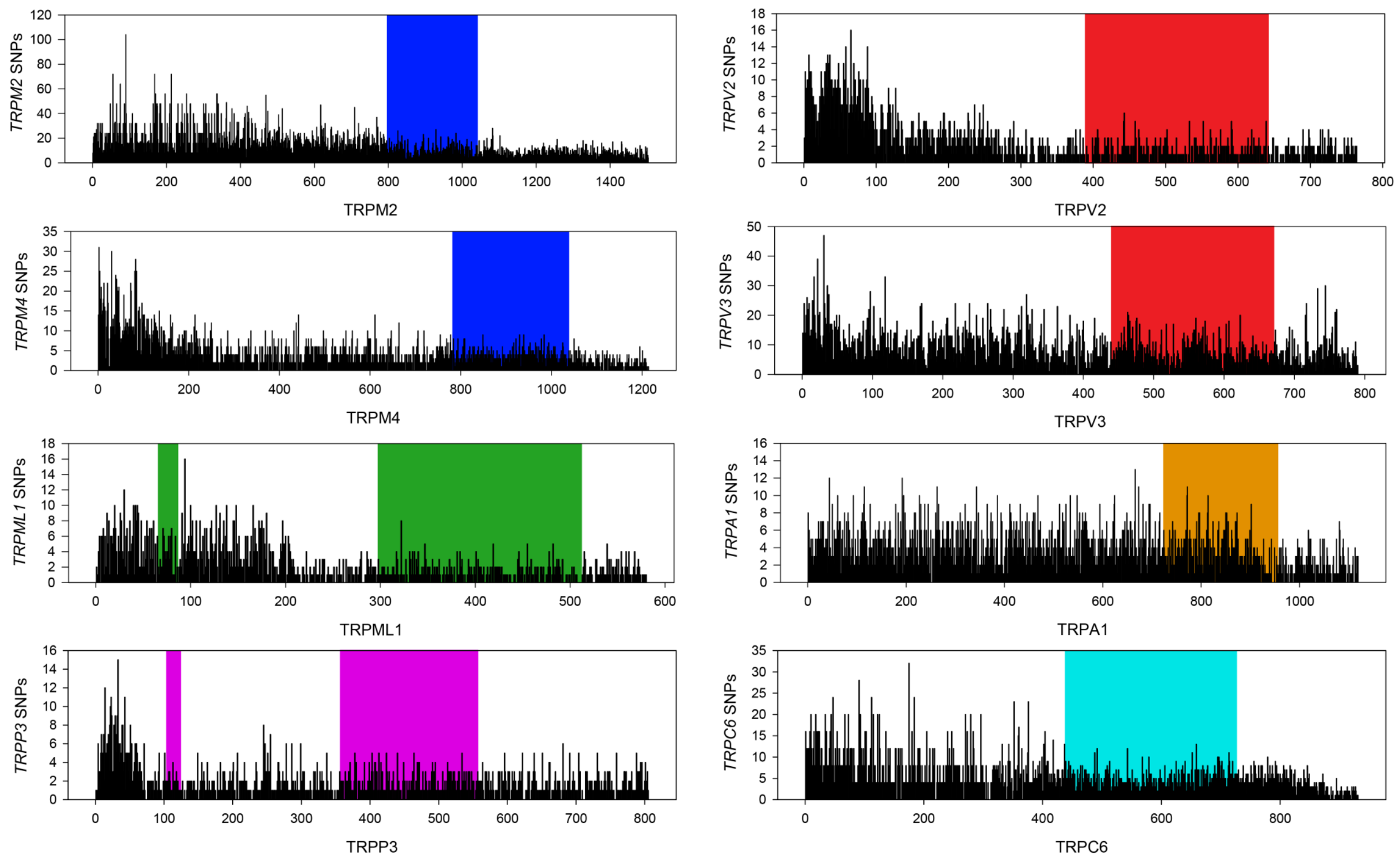

Figure S1. SNP frequencies by residue position in representative TRP channels. Exonic SNP data was downloaded from the Ensembl database (https://www.ensembl.org). Raw data was processed in Linux to count unique SNPs at each residue number. Color shading indicates transmembrane regions of the channels and corresponds to the colors of subfamilies shown in Figure 1. Note that TRPML and TRPP channels have an extended extracellular segment between the S1 helix and the rest of the transmembrane region. 